\title{
Mixed-mode dynamic stress intensity factors by variation technique with finite block method
}

\author{
H. Zheng ${ }^{1 *}$, Jingang Xiong ${ }^{1}$, Y. Yuan ${ }^{2}$ and P.H. Wen ${ }^{2 *}$ \\ ${ }^{I}$ School of Civil Engineering, Nanchang University, China \\ ${ }^{2}$ School of Engineering and Materials Science, Queen Mary University of London, UK
}

\begin{abstract}
The variational technique has been extended to calculate the dynamic stress intensity factors with the meshless finite block method in this paper. The Lagrange series is utilized to formulate the meshless approach in the normalized domain with coordinate mapping technique for practical problems. The stress intensity factors are evaluated by a variation of crack length for a mixed-mode fracture problem. Based on a static reference, the stress intensity factors in the Laplace space are determined with a boundary integral and domain integral. The Durbin inversion method is utilised to determine the time-dependent stress intensity factors. The applications of the proposed technique to two-dimensional fracture mechanics are demonstrated with three examples. Comparisons are made with the boundary element method and the meshless method in order to demonstrate the efficiency and accuracy of the proposed method.
\end{abstract}

Key words: Variational technique, meshless finite block method, mixed-mode stress intensity factors, Laplace transformation.

*Correspondence: Email: zhenghui @ncu.edu.cn (H. Zheng), p.h.wen@qmul.ac.uk (P.H. Wen) 


\section{Introduction}

In linear fracture mechanics, the focus is on the ability to determine the stress intensity factors at the crack tip under either static or dynamic loads. In 1976, Professor D.P. Rooke and D.J. Cartwright [1] for the first time documented several hundred analytical and numerical solutions in "A Compendium of Stress Intensity Factors". This documentation is considered as a significant handbook and database in the application of fracture mechanics. As the analytical solutions of the stress intensity factor are very rear in literature and publications, the development of numerical method is therefore important. Several numerical methods have been well developed to calculate the stress intensity factors for two- and three-dimensional problems, which include the finite element method (FEM, see Zienkiewicz [2]) and the boundary element method (BEM) by Brebbia [3], Balas et al [4]. One of the most successful numerical technique in fracture mechanics is the dual boundary element method (DBEM). This is due to its unique ability to deal with crack growth and fatigue life evaluation (see Aliabadi and Rooke [5]). Portela et al [6] demonstrated that DBEM for two-dimensional problems is efficient and accurate for the analysis of cracked structures. Mi and Aliabadi [7] developed the discontinuous quadrilateral quadratic elements for three-dimensional problems. A comprehensive review of the DBEM is given by Aliabadi [8]. Wen et al [9,10] proposed a contour integral technique and variational technique to determine two- and three-dimensional stress intensity factors using the indirect boundary element method (combination of the fictitious load method and displacement discontinuous method). The references were chosen as a crack embedded in an infinite plate and the derivatives of the traction and displacement on the boundary were determined numerically with a variation of crack area.

Although FEM and BEM have become very successful tools applied in engineering, the development of new advanced methods nowadays is still attractive in computational mechanics. Meshless approximations have received much interest since Nayroles et al [11] proposed the diffuse element method. Later, Belyschko et al [12] proposed element-free Galerkin method (EFGM) and Liu et al [13] developed the reproducing kernel particle methods. Recently, Atluri et al presented a family of Meshless methods, based on the Local weak Petrov-Galerkin formulation (MLPGs) for arbitrary partial differential equations [14-16] with moving leastsquare (MLS) approximation. MLPG is reported to provide a rational basis for constructing meshless methods with a greater degree of flexibility. Local Boundary Integral Equation (LBIE) 
with moving least square and polynomial radial basis function (RBF) was developed by Sladek et al $[17,18]$ for the boundary value problems in anisotropic non-homogeneous media, i.e. functionally graded materials. Both methods (MLPG and LBIE) are meshless due to the fact that no domain/boundary meshes are required. Based on the point collocation method, the meshless Finite Block Method (FBM) with mapping technique was proposed for elasticity problem by Wen and Cao [19]. One unique characteristic of the FBM is that the displacements and stresses are continuous along the interface between two blocks. Thereafter, Li and Wen [20] and $\mathrm{Li}$ et al [21,22] applied FBM to non-linear elasticity including heat conduction and contact problems in engineering successfully.

Wen et al $[9,10]$ developed the contour integral method and the variational technique firstly with the use of the boundary element method to evaluate stress intensity factors. In this paper, we present the numerical implementation of variational technique with the use of meshless finite block method for solving fracture dynamic problems. The accuracy has been illustrated by a rectangular sheet containing central/edge cracks subjected to static and dynamic loads. Excellent agreements with dual boundary element method and element free Galerkin method are achieved.

\section{Lagrange interpolation and mapping technique}

Based on the mapping technique, the meshless finite block method with Lagrange series interpolation was proposed in [22]. It is necessary to be introduced briefly in this section. For two dimensional problems, we use a set of nodes in the normalized domain at $P\left(\xi_{k}, \eta_{k}\right)$, $k=(j-1) \times N_{\xi}+i, i=1,2, \ldots, N_{\xi}$ and $j=1,2, \ldots, N_{\eta}$, where $N_{\alpha}$ indicates the number of nodes along either $\xi$ or $\eta$ axis. Function $u(Q)$ at $Q(\xi, \eta)$ can be approximated as

$$
u(Q)=\sum_{i=1}^{N_{\xi}} \sum_{j=1}^{N_{\eta}} F\left(\xi, \xi_{i}\right) G\left(\eta, \eta_{j}\right) u^{(k)}=\sum_{k=1}^{M} \phi_{k}(Q) u^{(k)}
$$

where

$$
F\left(\xi, \xi_{i}\right)=\prod_{\substack{m=1 \\ m \neq i}}^{N_{\xi}} \frac{\left(\xi-\xi_{m}\right)}{\left(\xi_{i}-\xi_{m}\right)}, G\left(\eta, \eta_{j}\right)=\prod_{\substack{n=1 \\ n \neq j}}^{N_{\eta}} \frac{\left(\eta-\eta_{n}\right)}{\left(\eta_{j}-\eta_{n}\right)}
$$

and the number of nodes in total is $M=N_{\xi} \times N_{\eta}$ for one block. We define the shape function as 


$$
\phi_{k}(Q)=F\left(\xi, \xi_{i}\right) G\left(\eta, \eta_{j}\right)=\prod_{\substack{m=1 \\ m \neq i}}^{N_{\xi}} \frac{\left(\xi-\xi_{m}\right)}{\left(\xi_{i}-\xi_{m}\right)} \prod_{\substack{n=1 \\ n \neq j}}^{N_{\eta}} \frac{\left(\eta-\eta_{n}\right)}{\left(\eta_{j}-\eta_{n}\right)}
$$

Therefore, we can derive the first order partial differential of shape function $\phi_{k}(Q)$ easily with respects to $Q$

$$
\frac{\partial \phi_{k}(Q)}{\partial \xi}=\frac{\partial F\left(\xi, \xi_{i}\right)}{\partial \xi} G\left(\eta, \eta_{j}\right), \frac{\partial \phi_{k}(Q)}{\partial \eta}=F\left(\xi, \xi_{i}\right) \frac{\partial G\left(\eta, \eta_{j}\right)}{\partial \eta}
$$

Let us arrange the nodal value of the first order partial differentials in vector form, in terms of the nodal value of function $u$, as

$$
\mathbf{u}_{, \alpha}=\mathbf{D}_{\alpha} \mathbf{u}, \mathbf{D}_{\alpha}=\left\{\phi_{k l, \alpha}\right\}_{M \times M}, \quad(k, l=1,2, \ldots, M ; \alpha=\xi, \eta),
$$

where

$$
\mathbf{u}_{, \alpha}=\left\{\frac{\partial u\left(P_{1}\right)}{\partial \alpha}, \frac{\partial u\left(P_{2}\right)}{\partial \alpha}, \ldots, \frac{\partial u\left(P_{M}\right)}{\partial \alpha}\right\}^{\mathrm{T}}, \mathbf{u}=\left\{u\left(P_{1}\right), u\left(P_{2}\right), \ldots, u\left(P_{M}\right)\right\}^{\mathrm{T}} .
$$

Therefore, the $L$-th order partial differentials

$$
u_{, m n}(\xi, \eta)=\frac{\partial^{m+n} u}{\partial \xi^{m} \partial \eta^{n}}, \quad m+n=L
$$

can be approximated by

$$
\mathbf{u}_{, m n} \approx \mathbf{D}_{\xi}^{m} \mathbf{D}_{\eta}^{n} \mathbf{u}
$$

Similar as the finite element method, a quadratic block with 8 seeds [19] is mapped into a normalized domain $(\xi, \eta)$ by the following shape functions, as shown in Figure 1(a), as

$$
\begin{aligned}
& N_{i}=\frac{1}{4}\left(1+\bar{\xi}_{i} \xi\right)\left(1+\bar{\eta}_{i} \eta\right)\left(\bar{\xi}_{i} \xi+\bar{\eta}_{i} \eta-1\right) \text { for } i=1,2,3,4 \\
& N_{i}=\frac{1}{2}\left(1-\xi^{2}\right)\left(1+\bar{\eta}_{i} \eta\right) \text { for } i=5,7 \\
& N_{i}=\frac{1}{2}\left(1-\eta^{2}\right)\left(1+\bar{\xi}_{i} \xi\right) \text { for } i=6,8
\end{aligned}
$$

where $\left(\bar{\xi}_{i}, \bar{\eta}_{i}\right), i=1,2, \ldots, 8$, are seed coordinates as shown in Figure 1(b). Then, we can transfer coordinate (mapping) for the real coordinate system into the normalized coordinate by 


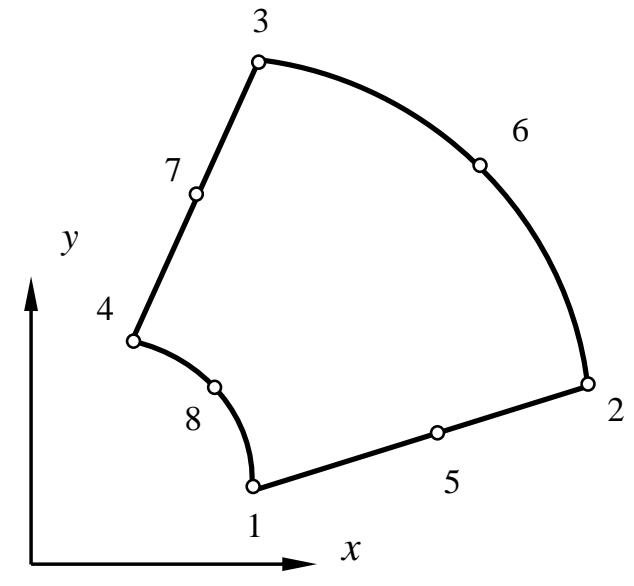

(a)

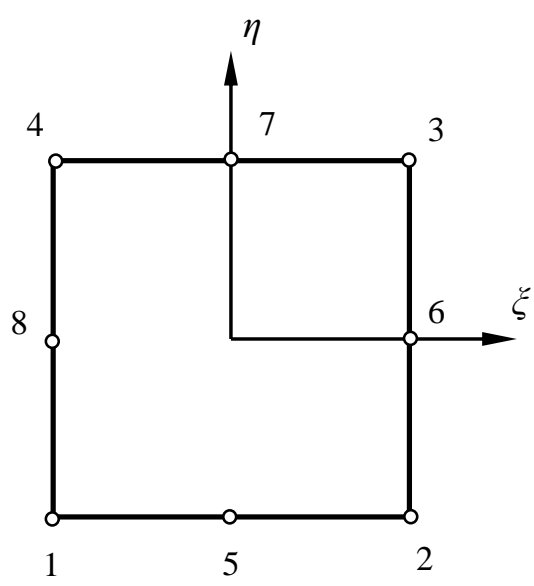

(b)

Figure 1. Mapping technique: (a) real domain; (b) normalized domain.

$$
x=\sum_{k=1}^{8} N_{k}(\xi, \eta) x_{k}, y=\sum_{k=1}^{8} N_{k}(\xi, \eta) y_{k} .
$$

The partial differentials of the function $u(x, y)$ are obtained by

$$
\frac{\partial u}{\partial x}=\frac{1}{J}\left(\beta_{11} \frac{\partial u}{\partial \xi}+\beta_{12} \frac{\partial u}{\partial \eta}\right), \frac{\partial u}{\partial y}=\frac{1}{J}\left(\beta_{21} \frac{\partial u}{\partial \xi}+\beta_{22} \frac{\partial u}{\partial \eta}\right)
$$

where

$$
J=\beta_{22} \beta_{11}-\beta_{21} \beta_{12}, \quad \beta_{11}=\frac{\partial y}{\partial \eta}, \beta_{12}=-\frac{\partial y}{\partial \xi}, \beta_{21}=-\frac{\partial x}{\partial \eta}, \beta_{22}=\frac{\partial x}{\partial \xi} .
$$

Let us arrange the first order partial differential matrices in the physical domain, in matrix form, as

$$
\begin{aligned}
& \mathbf{u}_{, x}=\boldsymbol{\Delta}_{11} \mathbf{U}_{\xi}+\boldsymbol{\Delta}_{12} \mathbf{U}_{\eta}=\left(\boldsymbol{\Delta}_{11} \mathbf{D}_{\xi}+\boldsymbol{\Delta}_{12} \mathbf{D}_{\eta}\right) \mathbf{u}=\mathbf{D}_{x} \mathbf{u}, \\
& \mathbf{u}_{, y}=\boldsymbol{\Delta}_{21} \mathbf{U}_{\xi}+\boldsymbol{\Delta}_{22} \mathbf{U}_{\eta}=\left(\boldsymbol{\Delta}_{21} \mathbf{D}_{\xi}+\boldsymbol{\Delta}_{22} \mathbf{D}_{\eta}\right) \mathbf{u}=\mathbf{D}_{y} \mathbf{u},
\end{aligned}
$$

in which

$$
\boldsymbol{\Delta}_{i j}=\left(\begin{array}{cccc}
\beta_{i j}^{(1)} / J^{(1)} & 0 & \ldots & 0 \\
0 & \beta_{i j}^{(2)} / J^{(2)} & \ldots & 0 \\
\ldots & \ldots & \ldots & \ldots \\
0 & 0 & \ldots & \beta_{i j}^{(M)} / J^{(M)}
\end{array}\right),
$$


$\beta_{i j}^{(k)} / J^{(k)}, k=1,2, \ldots, M$, at each collocation point $P\left(\xi_{k}, \eta_{k}\right)$. It is clear that the nodal values of the first order partial differentials in a real block can be determined in terms of the first order partial differential matrix in the normalized domain $|\xi| \leq 1 ;|\eta| \leq 1$.

\section{Finite block method in dynamic elasticity}

For two-dimensional dynamic elasticity, the governing equations, in terms of the stress, are

$$
\begin{array}{ll}
\frac{\partial \sigma_{x}}{\partial x}+\frac{\partial \tau_{x y}}{\partial y}=\rho \frac{\partial^{2} u}{\partial t^{2}} & \\
\frac{\partial \tau_{x y}}{\partial x}+\frac{\partial \sigma_{y}}{\partial y}=\rho \frac{\partial^{2} v}{\partial t^{2}} & (x, y) \in \Omega
\end{array}
$$

where $u$ and $v$ are displacements, $\rho$ indicates the mass density. For plane-strain case, we hold the relationship between stress and strain, by Hook's law, as

$$
\begin{aligned}
\sigma_{x} & =\frac{E(1-v)}{(1+v)(1-2 v)}\left(\frac{\partial u}{\partial x}+\frac{v}{1-v} \frac{\partial v}{\partial y}\right), \\
\sigma_{y} & =\frac{E(1-v)}{(1+v)(1-2 v)}\left(\frac{v}{1-v} \frac{\partial u}{\partial x}+\frac{\partial v}{\partial y}\right), \\
\tau_{x y} & =\frac{E}{2(1+v)}\left(\frac{\partial u}{\partial y}+\frac{\partial v}{\partial x}\right) .
\end{aligned}
$$

in which $E$ and $v$ are Yong's modulus and Poisson ratio respectively. The accelerations in (15)(16) should be omitted for the static case (or $\rho=0$ ). The boundary conditions are given, for $t>0$, by

$$
u(t)=u^{0}(t), \quad v(t)=v^{0}(t) \quad(x, y) \in \Gamma_{u},
$$

for displacement boundary $\Gamma_{u}$, and

$$
t_{x}(t)=t_{x}^{0}(t), \quad t_{y}(t)=t_{y}^{0}(t) \quad(x, y) \in \Gamma_{t},
$$

for traction boundary $\Gamma_{t}$. In addition, the initial conditions are specified in domain

$$
u(0)=U, \dot{u}(0)=U_{t}, v(0)=V, \quad \dot{v}(0)=V_{t} \quad(x, y) \in \Omega .
$$

Application of the Laplace transform over both sides of the equilibrium equations gives

$$
\frac{\partial \tilde{\sigma}_{x}}{\partial x}+\frac{\partial \tilde{\tau}_{x y}}{\partial y}=\rho\left(s^{2} \tilde{u}-s U-U_{t}\right),
$$


$\frac{\partial \tilde{\tau}_{x y}}{\partial x}+\frac{\partial \tilde{\sigma}_{y}}{\partial y}=\rho\left(s^{2} \bar{v}-s V-V_{t}\right) \quad(x, y) \in \Omega$,

where the Laplace transform of function $f(t)$ is defined

$\tilde{f}(s)=\int_{0}^{\infty} f(t) e^{-s t} d t$.

From (18)(19), the displacements and tractions in the Laplace domain satisfy:

$$
\begin{array}{lll}
\tilde{u}(x, y, s)=\tilde{u}^{0}(x, y, s), & \tilde{v}(x, y, s)=\tilde{v}^{0}(x, y, s) & (x, y) \in \Gamma_{u}, \\
\tilde{t}_{x}(x, y, s)=\tilde{t}_{x}^{0}(x, y, s), \tilde{t}_{y}(x, y, s)=\tilde{t}_{y}^{0}(x, y, s) & (x, y) \in \Gamma_{t} .
\end{array}
$$

Introducing the mapping technique with differential matrices in (13a) and (13b) gives

$$
\begin{aligned}
& \left(c_{1} \mathbf{D}_{x} \mathbf{D}_{x}+c_{2} \mathbf{D}_{y} \mathbf{D}_{y}-s^{2} \boldsymbol{\rho}\right) \tilde{\mathbf{u}}+c_{3} \mathbf{D}_{x} \mathbf{D}_{y} \tilde{\mathbf{v}}=-s \boldsymbol{\varphi}_{x}-\boldsymbol{\theta}_{x}, \\
& c_{3} \mathbf{D}_{x} \mathbf{D}_{y} \tilde{\mathbf{u}}+\left(c_{2} \mathbf{D}_{x} \mathbf{D}_{x}+c_{1} \mathbf{D}_{y} \mathbf{D}_{y}-s^{2} \boldsymbol{\rho}\right) \tilde{\mathbf{v}}=-s \boldsymbol{\varphi}_{y}-\boldsymbol{\theta}_{y},
\end{aligned}
$$

where the diagonal matrices: $\boldsymbol{\rho}=\operatorname{diag}[\rho], \boldsymbol{\varphi}_{x}=\operatorname{diag}[\rho U], \boldsymbol{\varphi}_{y}=\operatorname{diag}[\rho V], \boldsymbol{\theta}_{x}=\operatorname{diag}\left[\rho U_{t}\right]$, $\boldsymbol{\theta}_{y}=\operatorname{diag}\left[\rho V_{t}\right]$ and coefficients

$c_{1}=\frac{E(1-v)}{(1+v)(1-2 v)}, c_{2}=\frac{E}{2(1+v)}, c_{3}=\frac{E}{2(1+v)(1-2 v)}$.

Therefore, we can write the governing equations for each node in each block, in matrix form, as

$$
\mathbf{A}_{D}(s)(\tilde{\mathbf{u}}, \tilde{\mathbf{v}})^{\mathrm{T}}=\left(\tilde{\mathbf{b}}_{x}, \tilde{\mathbf{b}}_{y}\right)^{\mathrm{T}}
$$

where $\mathbf{A}_{D}(s)$ is a matrix with complex variable $s$ from $(24 \mathrm{a})(24 \mathrm{~b}), \quad \tilde{\mathbf{b}}_{x}=-s \boldsymbol{\varphi}_{x}-\boldsymbol{\theta}_{x}$ and $\tilde{\mathbf{b}}_{y}=-s \boldsymbol{\varphi}_{y}-\boldsymbol{\theta}_{y}$. In the same way, we derive the traction boundary conditions (23b), in matrix form, as

$$
\begin{aligned}
& \left(c_{1} \mathbf{D}_{x} n_{x}+c_{2} \mathbf{D}_{y} n_{y}\right) \tilde{\mathbf{u}}+\left(c_{2} \mathbf{D}_{x} n_{y}+c_{4} \mathbf{D}_{y} n_{x}\right) \tilde{\mathbf{v}}=\tilde{\mathbf{t}}_{x}^{0}, \\
& \left(c_{2} \mathbf{D}_{y} n_{x}+c_{4} \mathbf{D}_{x} n_{y}\right) \tilde{\mathbf{u}}+\left(c_{1} \mathbf{D}_{y} n_{y}+c_{2} \mathbf{D}_{x} n_{x}\right) \tilde{\mathbf{v}}=\tilde{\mathbf{t}}_{y}^{0},
\end{aligned}
$$

where

$$
c_{4}=\frac{v E}{(1+v)(1-2 v)} .
$$


Considering the governing equation in the domain for each block, connect conditions along the interface between two blocks, displacement boundary condition (23a) and traction boundary condition in (27), we may obtain the system equation as

$$
\mathbf{A}(s)(\tilde{\mathbf{u}}, \tilde{\mathbf{v}})^{\mathrm{T}}=\left(\tilde{\mathbf{f}}_{x}, \tilde{\mathbf{f}}_{y}\right)^{\mathrm{T}}
$$

where vectors $\left(\tilde{\mathbf{f}}_{x}, \tilde{\mathbf{f}}_{y}\right)$ consist the body forces in (26), tractions in (27) and displacements in (23a). Therefore, we are able to determine the nodal values of the displacement by solving algebraic system equations (29) in Laplace space.

Among several Laplace inverse algorithms, one of the simplest methods was proposed by Durbin [23] in 1975. By Durbin's method, the time-dependent function $f(t)$ can be approximated, with $(K+1)$ samples $\tilde{f}\left(s_{k}\right)$ in Laplace space at $s_{k}$, as

$$
f(t) \approx \frac{2 e^{\sigma / T}}{T}\left[-\frac{1}{2} \tilde{f}\left(s_{0}\right)+\sum_{k=0}^{K} \operatorname{Re}\left\{\tilde{f}\left(s_{k}\right) e^{2 \pi i k t / T}\right\},\right.
$$

where $s_{k}=(\sigma+2 \pi i k) / T, k=0,1, \ldots, K$. Again, the solution of the static case can be obtained simply by letting $\rho=0$ in the above numerical procedure.

\section{Variational technique in fracture mechanics}

Let us consider a variation $\delta a$ of crack length at crack tip $\mathbf{x}_{c}=\left(x_{c}, y_{c}\right)$ along the extension of crack surface with slant angle $\alpha$ (see Figure 2), the variation of crack tip coordinate $\delta \mathbf{x}_{c}=(\cos \alpha, \sin \alpha) \delta a$. Taking variation on both side of the governing equation (29) gives

$$
\frac{\delta \mathbf{A}(s)}{\delta a} \tilde{\mathbf{u}}+\mathbf{A}(s) \frac{\delta \widetilde{\mathbf{u}}}{\delta a}=0
$$

Thus, we have

$$
\frac{\delta \widetilde{\mathbf{u}}}{\delta a}=-\mathbf{A}^{-1}(s) \frac{\delta \mathbf{A}(s)}{\delta a} \tilde{\mathbf{u}}
$$

In the above variation equation, the derivative $\frac{\delta \mathbf{A}(s)}{\delta a}$ consists of the derivatives $\frac{\delta \Delta_{i j}}{\delta a}$ in (14) at each node. From (14), we have

$$
\frac{\delta \Delta_{i j}}{\delta a}=\frac{\delta\left(\beta_{i j} / J\right)}{\delta a}=\frac{1}{J^{2}}\left(J \frac{\delta \beta_{i j}}{\delta a}-\beta_{i j} \frac{\delta J}{\delta a}\right)
$$


where

$$
\begin{aligned}
& \frac{\delta J}{\delta a}=\frac{\delta \beta_{22}}{\delta a} \beta_{11}+\beta_{22} \frac{\delta \beta_{11}}{\delta a}-\frac{\delta \beta_{12}}{\delta a} \beta_{21}-\beta_{12} \frac{\delta \beta_{21}}{\delta a} \\
& \frac{\delta \beta_{11}}{\delta a}=\frac{\partial N_{n}^{L}}{\partial \eta} \sin \alpha, \beta_{12}=-\frac{\partial N_{n}^{L}}{\partial \xi} \sin \alpha, \beta_{21}=-\frac{\partial N_{n}^{L}}{\partial \eta} \cos \alpha, \beta_{22}=\frac{\partial N_{n}^{L}}{\partial \xi} \cos \alpha .
\end{aligned}
$$

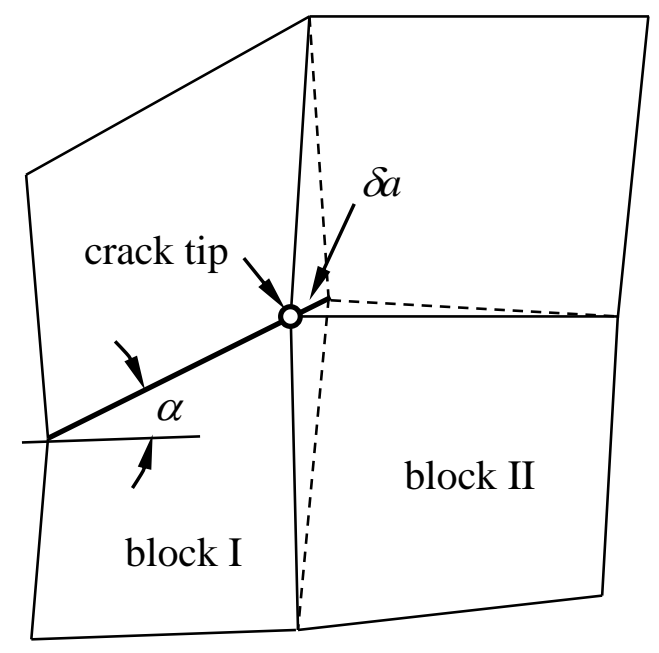

Figure 2. Blocks surround the crack tip with variation of crack length.

in which $N_{n}^{L}$ denotes shape function from (9), $n$ is the seed number corresponding to the crack tip in block $L$. In order to determine the stress intensity factors, we need the variations of displacement and traction need. Let us consider a static problem as a reference $(u, v)$ with the same configuration of a cracked plate and zero body forces. Apply the reciprocal theory gives

$$
\int_{\Gamma}\left(u \tilde{t}_{x}^{0}+v \tilde{t}_{y}^{0}-t_{x} \tilde{u}^{0}-t_{y} \tilde{v}^{0}\right) d \Gamma-\rho s^{2} \int_{\Omega}(u \tilde{u}+v \tilde{v}) d \Omega=0, \quad \Gamma=\Gamma_{u}+\Gamma_{t} .
$$

Applying reciprocal theory again with a variation of crack length $\delta a$ to the reference gives

$$
\begin{aligned}
K_{\mathrm{I}}^{\text {static }} \tilde{K}_{\mathrm{I}}+K_{\mathrm{II}}^{\mathrm{static}} \tilde{K}_{\mathrm{II}} & =\frac{(1-v) E}{2(1+v)(1-2 v) \delta a}\left[\int_{\Gamma}\left((u+\delta u) \tilde{t}_{x}^{0}+(v+\delta v) \tilde{t}_{y}^{0}-\left(t_{x}+\delta t_{x}\right) \tilde{u}^{0}-\left(t_{y}+\delta t_{y}\right) \tilde{v}^{0}\right) d \Gamma\right. \\
& \left.-\rho s^{2} \int_{\Omega}((u+\delta u) \tilde{u}+(v+\delta v) \tilde{v}) d \Omega\right]
\end{aligned}
$$

Therefore, we have 


$$
\begin{aligned}
K_{\mathrm{I}}^{\text {static }} \tilde{K}_{\mathrm{I}}+K_{\mathrm{II}}^{\text {static }} \tilde{K}_{\mathrm{II}} & =\frac{(1-v) E}{2(1+v)(1-2 v) \delta a}\left[\int_{\Gamma}\left(\delta u \tilde{t}_{x}^{0}+\delta v \tilde{t}_{y}^{0}-\delta t_{x} \tilde{u}^{0}-\delta t_{y} \tilde{v}^{0}\right) d \Gamma-\right. \\
& \left.-\rho s^{2} \int_{\Omega}(\delta u \tilde{u}+\delta v \tilde{v}) d \Omega\right] .
\end{aligned}
$$

On the boundary, we hold

$$
\begin{array}{ll}
\delta u=\delta v=0 & (x, y) \in \Gamma_{u}, \\
\delta t_{x}=\delta t_{y}=0 & (x, y) \in \Gamma_{t} .
\end{array}
$$

Therefore, the mixed-mode transformed stress intensity factors, for a plane strain problem, yields from (38)

$$
\begin{aligned}
\tilde{K}_{\mathrm{I}}(s) & =\frac{(1-v) E}{2(1+v)(1-2 v) K_{\mathrm{I}}^{\text {static }}\left(1+\varphi \varphi^{*}\right)}\left[\int_{\Gamma_{t}}\left(\frac{\delta u}{\delta a} \tilde{t}_{x}^{0}+\frac{\delta v}{\delta a} \tilde{t}_{y}^{0}\right) d \Gamma-\int_{\Gamma_{u}}\left(\frac{\delta t_{x}}{\delta a} \tilde{u}^{0}+\frac{\delta t_{y}}{\delta a} \tilde{v}^{0}\right) d \Gamma\right. \\
& \left.-\rho s^{2} \int_{\Omega}\left(\frac{\delta u}{\delta a} \tilde{u}+\frac{\delta v}{\delta a} \tilde{v}\right) d \Omega\right], \\
\tilde{K}_{\mathrm{II}}(s) & =\varphi^{*} \tilde{K}_{\mathrm{I}}(s),
\end{aligned}
$$

in which $\varphi^{*} \approx \frac{\Delta \tilde{u}_{\tau}}{\Delta \tilde{u}_{n}}, \Delta \tilde{u}_{\tau}$ and $\Delta \tilde{u}_{n}$ are transformed tangential and normal discontinuous displacements (COD) near crack tip. We can use (40) directly to determine static stress intensity factors

$$
\begin{aligned}
K_{\mathrm{I}}^{\text {static }} & =\left(\frac{E(1-v)}{2(1-v)(1-2 v)\left(1+\varphi^{2}\right)}\left[\int_{\Gamma_{t}}\left(\frac{\delta u}{\delta a} t_{x}^{0}+\frac{\delta v}{\delta a} t_{y}^{0}\right) d \Gamma-\int_{\Gamma_{u}}\left(\frac{\delta t_{x}}{\delta a} u^{0}+\frac{\delta t_{y}}{\delta a} v^{0}\right) d \Gamma\right]\right)^{1 / 2}, \\
K_{\mathrm{II}}^{\text {static }} & =\varphi K_{\mathrm{I}}^{\text {static }},
\end{aligned}
$$

where $\varphi \approx \frac{\Delta u_{\tau}}{\Delta u_{n}}, \Delta u_{\tau}$ and $\Delta u_{n}$ are static tangential and normal discontinuous displacements near crack tip.

\section{Examples}

In the Durbin method, we have two free parameters $\sigma$ and $T$, which affect the accuracy slightly [23]. In the following examples, we take $\sigma=5 / t_{0}$ and $T / t_{0}=20$, where time unit $t_{0}=w / c_{d}, w$ is a characteristic length such as the width of plate and the velocity of the 
longitudinal wave $c_{d}=\sqrt{E(1-v) / \rho(1+v)(1-2 v)}$. The number of samples in Laplace space is $K+1$ and $K$ is taken to 25. Apart from a boundary integral in (40), a domain integral needs to be calculated. As there is no singularity in these integrals, we evaluate all integrals by any regular integral scheme. In the following examples, the square domain integration in the normalized domain $(\xi, \eta)$ is performed by dividing the square into $10 \times 10$ cells with $4 \times 4$ Gauss points for each cell. The location of the node along the two axes in the normalized domain is selected as

$$
\xi_{i}=-\cos \frac{\pi(i-1)}{N_{\xi}-1}, i=1,2, \ldots, N_{\xi}, \quad \eta_{j}=-\cos \frac{\pi(j-1)}{N_{\eta}-1}, j=1,2, \ldots, N_{\eta}
$$

and the number of nodes in total $M=N_{\xi} \times N_{\eta}$ for each block. To determine the tangential and normal discontinuous displacements near the crack tip, we consider the third node from the crack tip in all examples by use of COD.

\subsection{Central/edge cracks in a rectangular sheet under uniform static load $\sigma_{0}$}

For the sake of simplicity, to validate the degrees of accuracy and efficiency by the use of the variational technique, we observe an embedded crack in a rectangular sheet under static load firstly shown in Figure 3(a). Let us suppose a rectangular plate of width $2 w$ and height $2 h$ with a centred crack of $2 a$ is subjected to a uniform load $\sigma_{0}$ on the top and bottom. Due to the symmetry of the problem, a quarter of the plate is modelled as shown in Figure 3(b) with only two blocks. Yong's modulus is taken as a unit and Poisson's ratio $v=0.3$. The normalize static Stress Intensity Factors (SIFs) $K_{\mathrm{I}}^{\text {static }} / \sigma_{0} \sqrt{\pi a}$ by both variational technique and COD are listed in Table 1 versus ratios $a / w$ and $h / w$. We take the node density $N_{\xi}=N_{\eta}=13$ and define the average relative errors as

$$
\varepsilon(\%)=\frac{1}{N_{0}} \sum_{i=1}^{N_{0}}\left|K_{\mathrm{I}}^{\text {static }}\left(a_{i}\right)-K_{\mathrm{I}}^{0}\left(a_{i}\right)\right| / K_{\mathrm{I}}^{0}\left(a_{i}\right) .
$$

where $N_{0}$ is the number of crack length $a_{i}, K_{\mathrm{I}}^{0}\left(a_{i}\right)$ is from handbook [1]. It is clear that the average relative errors are $1.1 \%$ and $0.7 \%$ by using the variational method and $1.3 \%$ and $4.8 \%$ by COD method when $h / w=1$ and 0.5 respectively. The same conclusion can be found for a double edge cracked sheet from Table 2. To study the convergence, we present normalized 
SIFs in Table 3 with different node densities for a central crack, where $N_{\xi}=N_{\eta}=N$, $a / w=0.5$ and $h / w=0.5$. Excellent convergence can be obtained for both the variational method and COD method.

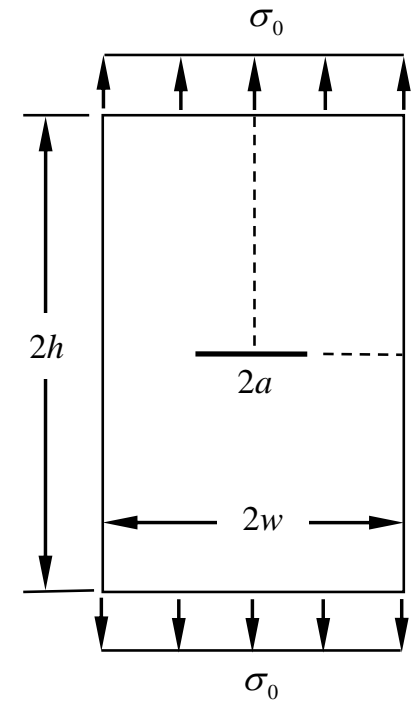

(a)

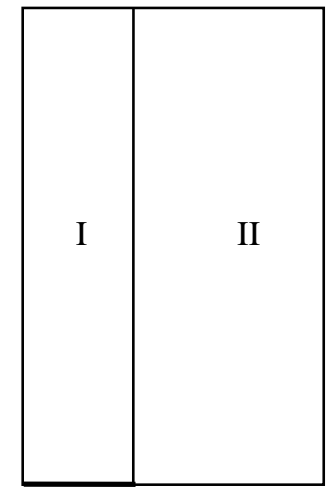

(b)

Figure 3. Plate with a central crack: (a) geometry; (b) quarter of the plate with two blocks.

Table 1. Normalised stress intensity factors $K_{\mathrm{I}} / \sigma_{0} \sqrt{\pi a}$ for central crack.

\begin{tabular}{ccccccc}
\hline \multirow{2}{*}{$a / w$} & \multicolumn{3}{c}{$h / w=1$} & \multicolumn{3}{c}{$h / w=0.5$} \\
\cline { 2 - 7 } & Variation & COD & {$[1]$} & Variation & COD & {$[1]$} \\
\hline 0.2 & 1.1554 & 1.0570 & 1.10 & 1.1665 & 1.1949 & 1.17 \\
0.3 & 1.1169 & 1.1108 & 1.12 & 1.3598 & 1.3794 & 1.35 \\
0.4 & 1.2057 & 1.1928 & 1.20 & 1.6160 & 1.6497 & 1.60 \\
0.5 & 1.3218 & 1.3060 & 1.32 & 1.9520 & 2.0227 & 1.93 \\
0.6 & 1.4671 & 1.4541 & 1.46 & 2.4082 & 2.5522 & 2.40 \\
0.7 & 1.6602 & 1.6552 & 1.67 & 3.0248 & 3.3235 & 3.00 \\
0.8 & 1.9686 & 1.9673 & ---- & 3.7611 & 4.4009 & ---- \\
$\varepsilon \%$ & $1.1 \%$ & $1.3 \%$ & & $0.7 \%$ & $4.8 \%$ & \\
\hline
\end{tabular}


Table 2. Normalised stress intensity factors $K_{\mathrm{I}} / \sigma_{0} \sqrt{\pi a}$ for edge crack.

\begin{tabular}{cccccc}
\hline \multirow{2}{*}{$a / w$} & \multicolumn{3}{c}{$h / w=1$} & \multicolumn{3}{c}{$h / w=0.5$} \\
\cline { 2 - 6 } & Variation & COD & {$[1]$} & Variation & COD \\
\hline 0.2 & 1.1841 & 1.2101 & 1.15 & 1.4677 & 1.5409 \\
0.3 & 1.2234 & 1.2483 & 1.19 & 1.7800 & 1.8536 \\
0.4 & 1.2758 & 1.2907 & 1.23 & 2.1145 & 2.2153 \\
0.5 & 1.3241 & 1.3346 & 1.28 & 2.4157 & 2.5644 \\
0.6 & 1.3755 & 1.3832 & 1.33 & 2.5952 & 2.8083 \\
0.7 & 1.4552 & 1.4575 & 1.41 & 2.5629 & 2.8416 \\
0.8 & 1.6268 & 1.6119 & ---- & 2.3684 & 2.6756 \\
$\varepsilon \%$ & $3.2 \%$ & $4.4 \%$ & & & \\
\hline
\end{tabular}

Table 3. SIFs versus node density

\begin{tabular}{ccc}
\hline$N$ & Variation & COD \\
\hline 5 & 1.67071 & 1.95372 \\
9 & 1.92801 & 2.04998 \\
13 & 1.95203 & 2.02266 \\
17 & 1.95906 & 2.00611 \\
\hline
\end{tabular}

\subsection{A central crack embedded in a rectangular plate under dynamic tension}

Let us consider a rectangular plate of width $2 w$ and length $2 h$ with a central crack of length $2 a$ in Example 5.1. subjected to a dynamic uniform tension load $\sigma_{0} H(t)$ on the top and bottom, here $H(t)$ is the Heaviside step function. Let Poisson ratio $v=0.3$, ratio $a / w=0.5$ and Young's modulus be one unit. Two ratios of height and width are considered, i.e. $h / w=1$, $h / w=0.5$, with node density $N_{\xi}=N_{\eta}=13$. Figures 4 and 5 show normalized dynamic stress intensity factors $K_{\mathrm{I}}(t) / \sigma_{0} \sqrt{\pi a}$ for different ratios of height and width respectively. The achieved results are compared with indirect Boundary Element Method (BEM) [24] and the element free Galerkin method (EFM) [25]. In general, the maximum value of dynamic stress intensity factor for each case is found to be twice that of the static value [24]. As the solution by BEM is assumed to be more accurate, it can be concluded that the degree of accuracy by 
variational technique is higher than that by EFM [25]. In addition, a comprehensive review on the selections of the two free parameters $\sigma$ and $T$ in Durbin inverse method can be found in [23], therefore, it is not necessary to discuss here.

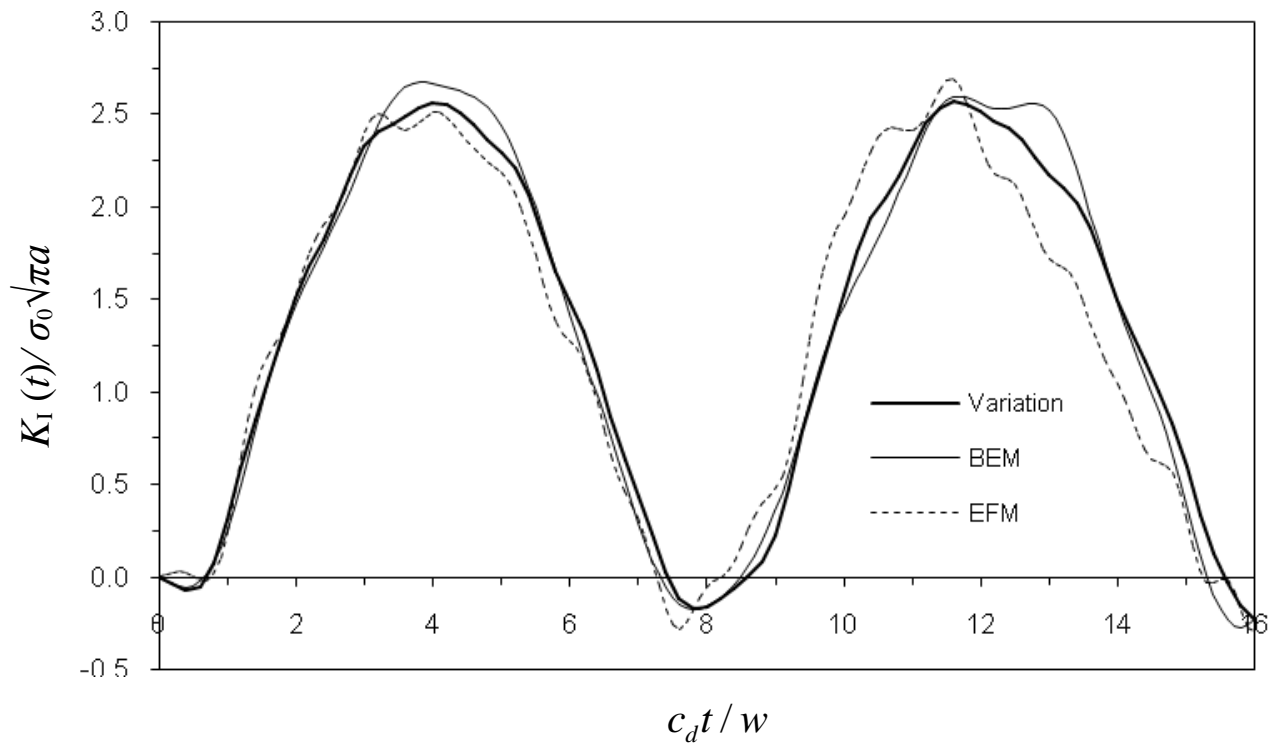

Figure 4. Normalized dynamic stress intensity factor $K_{\mathrm{I}}(t) / \sigma_{0} \sqrt{\pi a}$ for a central crack under uniform tension load $\sigma_{0} H(t)$ while $a / w=0.5, h / w=1$ and $v=0.3$.

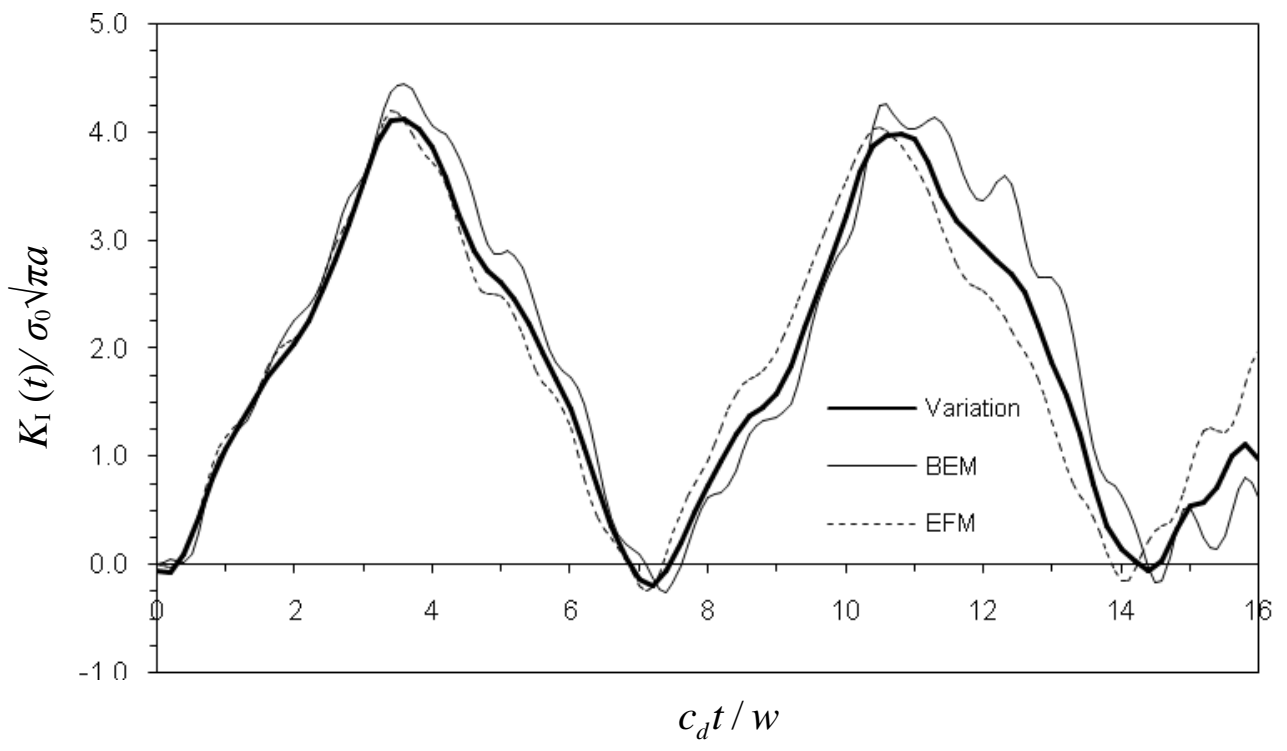

Figure 5. Normalized dynamic stress intensity factor $K_{\mathrm{I}}(t) / \sigma_{0} \sqrt{\pi a}$ for a central crack under uniform tension load $\sigma_{0} H(t)$ while $a / w=0.5, h / w=0.5$ and $v=0.3$. 


\subsection{Rectangular plate with an inclined edge crack under dynamic load}

Let us consider a rectangular panel with a slanted edge crack at angle $\alpha$ with three simply supported edges loaded by a Heaviside tensile load $\sigma_{0} H(t)$ on the top surface. Figures 6(a)(b) show the geometry of cracked plate with four blocks to construct the computational model. The panel is of the following material properties [26]: shear modulus $\mu=29.4 \times 10^{9} \mathrm{~Pa}$; density $\rho=2450 \mathrm{kgm}^{-3}$; Poisson's ratio $v=0.286$. We observe two cases: (a) $\alpha=45^{\circ}, a=22.63 \mathrm{~mm}$; (b) $\alpha=0^{0}, a=16 \mathrm{~mm}$. Normalized mixed-mode $\operatorname{SIFs} K_{\mathrm{I}}(t) / \sigma_{0} \sqrt{\pi a}$ and $K_{\mathrm{II}}(t) / \sigma_{0} \sqrt{\pi a}$ versus real time in $\mu s$ are shown in Figure 7 . The solutions by using dual boundary element method [26] are also plotted in the same figure for comparison. We can see clearly that these solutions by different approaches are matched very well. To observe SIFs with a longer period of time, i.e. in the region $0<c_{d} t / w<10$, we present the time-dependent SIFs for different cases in Figures 8 and 9. The results by the meshless method with enriched radial basis functions [27] are also presented in the same figures for comparison. As expected, the absolute value of mode II SIF $K_{\mathrm{II}}(t)$ is much smaller than $K_{\mathrm{I}}(t)$ when $\alpha=0^{0}$. The numerical solutions show the reasonable agreement between these two methods.

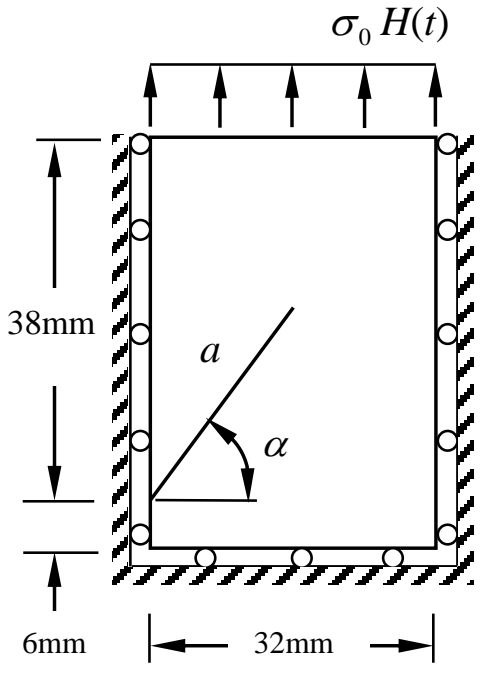

(a)

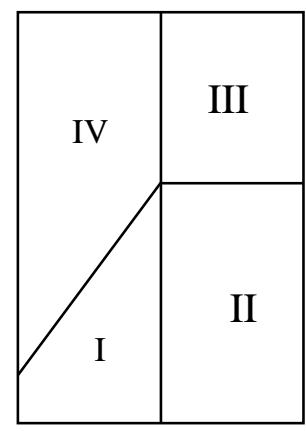

(b)

Figure 6. Slant edge crack in plate: (a) Geometry and dynamic load; (b) modelling with four blocks. 


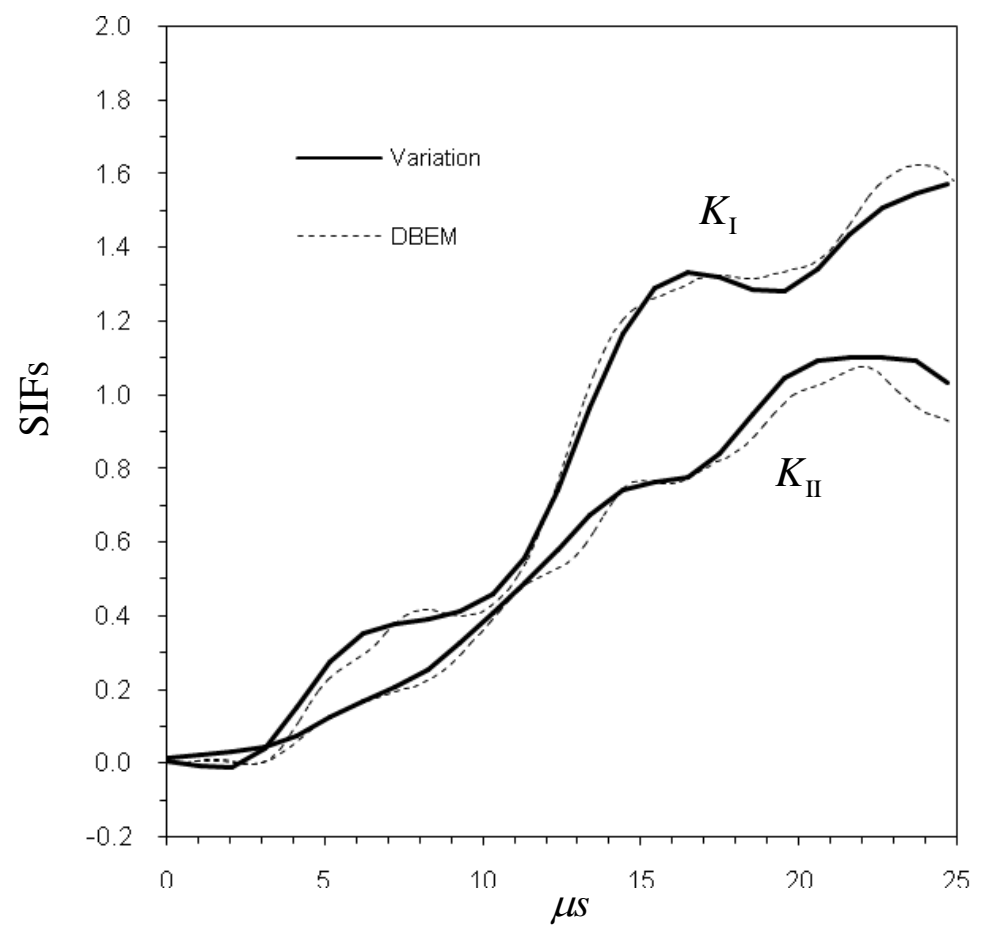

Figure 7. Normalized dynamic stress intensity factor $K_{\mathrm{I}}(t) / \sigma_{0} \sqrt{\pi a}$ by variational technique and dual boundary element method.

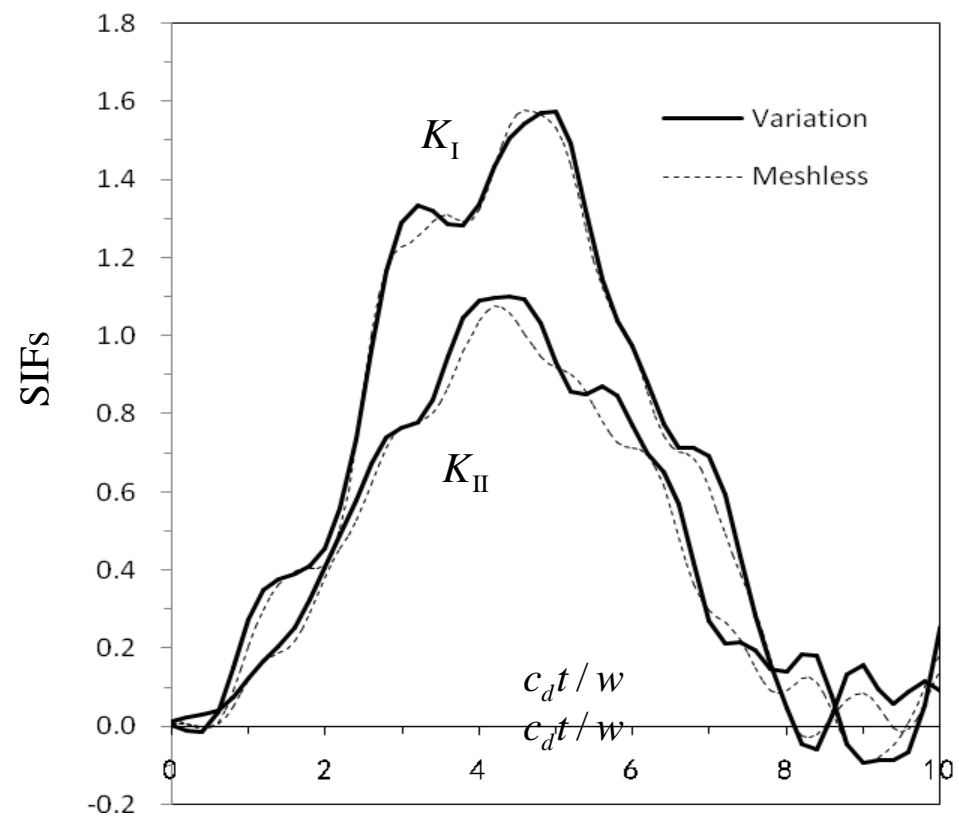

Figure 8. Normalized dynamic stress intensity factor $K_{\mathrm{I}}(t) / \sigma_{0} \sqrt{\pi a}$ by variational technique and meshless method versus normalized time $c_{d} t / w$ when $\alpha=45^{0}$. 


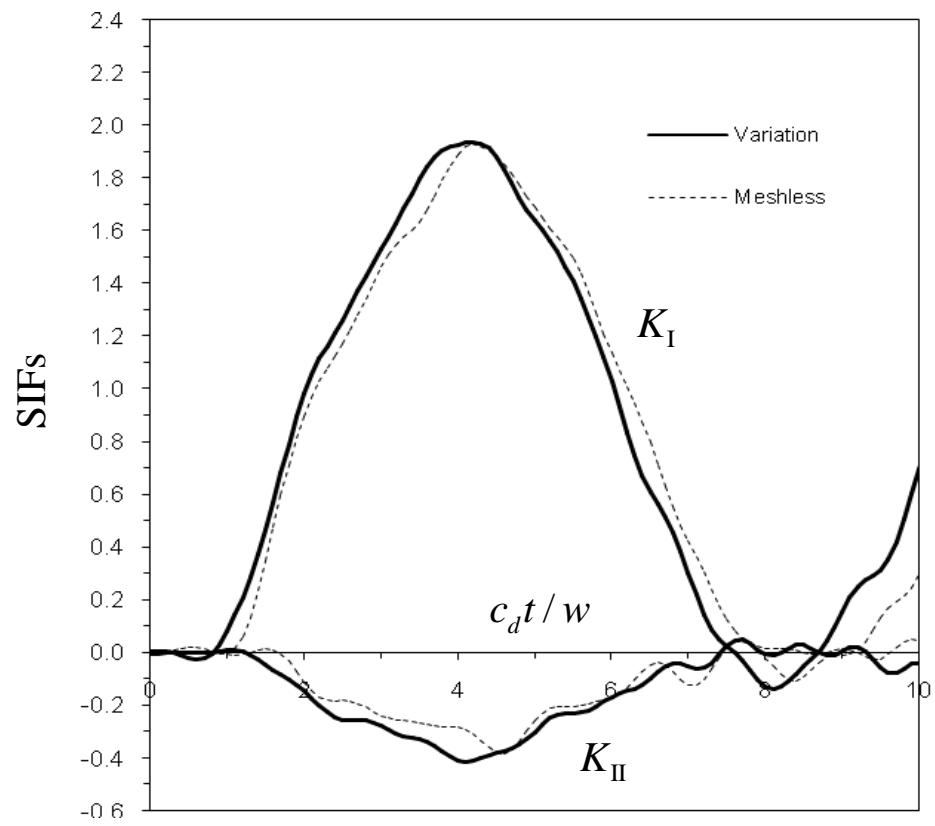

Figure 9. Normalized dynamic stress intensity factor $K_{\mathrm{I}}(t) / \sigma_{0} \sqrt{\pi a}$ by variational technique and meshless method versus normalized time $c_{d} t / w$ when $\alpha=0^{0}$.

\section{Conclusion}

The variational technique by the use of meshless finite block method in fracture mechanics was investigated in this paper. In order to determine the dynamic stress intensity factors, a static reference was introduced for different Laplace parameters in the transformed space. We evaluated the transformed stress intensity factors by using the reciprocal theory with a regular boundary integral and domain integral. The time-dependent variables were obtained by Durbin inverse technique. The degree of the accuracy and convergence of the proposed technique was demonstrated by three examples. We can make following conclusions can be made:

(1) The computational effort is reduced by using few blocks to discrete the cracked panel and convergence of the solution can be achieved;

(2) The variational technique with FBM is valid to deal with dynamic fracture problem with a higher degree of accuracy than COD method and EFM; 
(3) Reference can be chosen as a static case to save CPU time;

(4) Reasonable accurate solutions in the real time domain can be achieved by fewer samples in the Laplace space;

(5) The variational technique can be easily extended to three-dimensional elasticity, functionally graded material and plate bending crack problems.

\section{References}

[1] Rooke DP, Cartwright DJ. A Compendium of Stress Intensity Factors. HMSO, London, 1976.

[2] Zienkiewicz OC. The Finite Element Method. McGraw-Hill, New York, 1971.

[3] Brebbia CA. The Boundary Element Method for Engineers. Wiley-Blackwell, 1984.

[4] Balas J, Sladek J, Sladek V. Stress Analysis by Boundary Element Method. Elsevier, Amsterdam, 1989.

[5] Aliabadi MH, Rooke DP. Numerical Fracture Mechanics, Computational Mechanics Publications. Southampton and Kluwer Academic Publications, Dordrecht, 1991.

[6] Portela A, Aliabadi MH, Rooke DP. The dual boundary element method: effective implementation for crack problems. Int J Numer Meth Engng 1992; 33: 1269-1287.

[7] Mi Y, Aliabadi MH. Dual boundary element method for three-dimensional fracture mechanics analysis. Engng Anal Boundary Elements 1992; 10: 161-171.

[8] Aliabadi MH. The Boundary Element Method Vol2: Applications in Solids and Structures. John Wiley and Sons, 2002.

[9] Wen PH, Aliabadi MH, Rooke DP. A contour integral method for dynamic stress intensity factors. Theore Appl Fract Mech 1997; 27: 29- 41.

[10] Wen PH, Aliabadi MH, Rooke DP. A variational technique for boundary element analysis of 3D fracture mechanics weight functions: static. Int J Numer Meth Engng 1998; 42: 14091423.

[11] Nayroles B, Touzot G, Villon P. Generalizing the finite element method: diffuse approximation and diffuse elements. Computational Mechanics 1992; 10: 307-318.

[12] Belytschko T, Lu YY, Gu L. Element-free Galerkin method. Int J Numerical Methods in Engineering 1994;37: 229-256. 
[13] Liu WK, Jun S, Zhang Y. Reproducing kernel particle methods. Int J Numerical Methods in Engineering 1995;20: 1081-1106.

[14] Atluri SN, Zhu T. A new meshless local Peyrov-Galerkin (MLPG) approach to nonlinear problems in computational modelling and simulation. Comput Model Simul Engng 1998;3: 187-196.

[15] Atluri SN, Zhu T. The meshlesss local Peyrov-Galerkin (MLPG) approach for solving problems in elasto-statics. Comput Mech 1999;25: 169-179.

[16] Atluri SN. The Meshless Method (MLPG) for Domain and BIE Discretizations. Forsyth, GA, USA, Tech Science Press, 2004.

[17] Sladek J, Sladek V, Wen PH, Aliabadi MH. Meshless Local Petrov-Galerkin (MLPG) method for shear deformable shells analysis. Computer Modeling in Engineering \& Sciences 2006; 13: 103-117.

[18] Sladek V, Sladek J, Tanaka M, Zhang Ch. Local integral equation method for potential problems in functionally graded anisotropic materials. Engng Analy with Boundary Elements 2005; 29: 829-843.

[19] Wen PH, Cao P, Korakianitis T. Finite Block Method in elasticity. Engineering Analysis with Boundary Elements 2014; 46: 116-125.

[20] Li M, Wen PH. Finite block method for transient heat conduction analysis in functionally graded media. International Journal for Numerical Methods in Engineering 2014; 99(5): 372390.

[21] Li M, Lei M, Munjiza A, Wen PH. Frictional contact analysis of functionally graded materials with Lagrange finite block method. International Journal for Numerical Methods in Engineering 2015; 103(6): 391-412.

[22] Li M, Meng LX, Hinneh P, Wen PH. Finite block method for interface cracks. Engineering Fracture Mechanics 2016; 156: 25-40.

[23] Durbin F. Numerical inversion of Laplace transforms: an efficient improvement to Dubner and Abate's method. The Computer J 1975; 17: 371-376.

[24] Wen PH. Dynamic Fracture Mechanics: Displacement Discontinuity Method. Computational Mechanics Publications, Southampton UK and Boston USA, 1996.

[25] Wen PH, Aliabadi MH. A variational approach for evaluation of stress intensity factors using the element free Galerkin method. Int J Solids and Structures 2011;48: 1171-1179. 
[26] Fedelinski P, Aliabadi MH, Rooke DP. The Laplace transform DBEM for mixed-mode dynamic crack analysis. Computers \& Structures 1996;59(6): 1021-1031.

[27] Wen PH, Aliabadi MH. Meshless method with enriched radial basis functions for fracture mechanics. Structural Durability \& Health Monitoring 2007; 3 (2): 107-119. 